\title{
Cartesian Cut-Cell Method with Local Grid Refinement for Wave Computations
}

\author{
Mihaela Popescu * \\ Department of Process and Technology, \\ SINTEF Material and Chemistry, Trondheim, Norway, 7465 \\ Cheng-Feng Tai $^{\dagger}$ \\ Department of Mechanical and Aerospace Engineering \\ University of Florida, Gainesville, FL 32611 \\ and \\ Wei Shyy ${ }^{\ddagger}$ \\ Department of Aerospace Engineering \\ University of Michigan, Ann Arbor, MI 48109
}

\begin{abstract}
Sound generation from a vibrating circular piston is a classical acoustic problem. The goal of this paper is to simulate numerically the sound radiation produced by oscillating baffled pistons, using both linear and nonlinear model, and to consider the interplay between wave propagation and geometric complexities. The linear solution, based on the linear Euler equations, will be compared to the Rayleigh integral approximation. The nonlinear solution, based on the Navier-Stokes equations, will be compared against linear model for low speed (less than 0.01 of sound speed). A main practical interest in this problem is to capture the behavior of the waves resulting from the source pistons with other solid objects or waves. The wave's properties in terms of frequency, amplitude and wavenumber are influenced by the initial frequencies and coordinates of the pistons, and the geometry. The wave equations in Cartesian coordinate with cut-cell and local grid refinement technique are employed along with the Optimized Prefactored Compact finite volume (OPC-fv) scheme for spatial discretization, the Low-Dispersion Low-Dissipation Runge-Kutta (LDDRK) scheme for time discretization. Problems for the waves around different geometries, and with varied frequencies and amplitudes are considered and presented.
\end{abstract}

\section{Nomenclature}

$\begin{array}{lll}\mathrm{A} & = & \text { area } \\ \mathrm{CFL} & = & \text { Courant-Friedrichs-Lewy number } \rightarrow \mathrm{c} \Delta \mathrm{t} / \mathrm{min}(\Delta \mathrm{x}, \Delta \mathrm{y}) \\ D_{i}^{F}, D_{i}^{B} & = & \text { forward and backward derivative operators at the point } i \\ D(\theta) & = & \text { directivity factor } \\ D & = & \text { width of perfect matched layer } \\ K^{(\mathrm{i})} & = & \text { computed function at i in the Runge-Kutta time integration } \\ \mathrm{LDDRK} & = & \text { Low-Dissipation and Low-Dispersion Runge-Kutta schemes } \\ \text { OPC } & = & \text { Optimized Prefactored Compact scheme } \\ \mathrm{PML} & = & \text { Perfect Matched Layer } \\ R & = & \text { radial distance from origin } \\ a & = & \text { radius of the piston } \\ c & = & \text { speed of sound } \\ d & = & \text { distance between center of pistons } \\ \mathrm{fd} & = & \text { finite difference } \\ \mathrm{fv} & = & \text { finite volume }\end{array}$

\footnotetext{
* Post Doc, Department of Process and Technology, Sem Sælands vei 2 a, Trondheim, AIAA Member.

${ }^{\dagger}$ Ph.D. Student, Department of Mechanical and Aerospace Engineering, AIAA Member.

‡ Clarence L. "Kelly” Johnson Collegiate Professor and Chair, Fellow AIAA 


$\begin{array}{lll}k & = & \text { wavenumber } \\ k a & = & \text { Helmholtz number } \\ p & = & \text { pressure } \\ t & = & \text { time } \\ u & = & \text { Particle velocity } \\ u^{(m)} & = & \text { the value of function u in the stage m in the Runge-Kutta time integration } \\ u_{i}^{B e}, u_{i}^{F e} & = & \text { forward, and backward operator computed on east face in the cell i } \\ u_{i}^{B w}, u_{i}^{F w} & = & \text { forward, and backward operator computed on west face in the cell i } \\ u_{i}{ }^{F} & = & \text { the value of parameter u on face e, in cell i } \\ u_{i}{ }^{w} & = & \text { the value of parameter u on face w, in cell i } \\ u^{n} & = & \text { the value of function u in the } \mathrm{n} \text { iteration, it is related to time integration } \\ x, y & = & \text { spatial coordinates } \\ \Delta x, \Delta y, \Delta t & = & \text { grid size in space,r, } \mathrm{x} \text { and y directions, and time step size } \\ \eta_{k}, \beta_{k}, \gamma_{k}, a_{k}, b_{k}, & = & \text { coefficients used in generic stencils for forward and backward derivatives of OPC } \\ c_{k}, d_{k}(k=F \text { or } B) & & \text { scheme } \\ \varepsilon & = & \text { error } \\ \lambda & = & \text { wavelength } \\ \theta & = & \text { Angle between } R \text { and axis } O x \\ \rho & = & \text { density } \\ \sigma_{x}, \sigma_{y} & = & \text { absorbing coefficients of PML domain } \\ \tau & = & \text { shear stress tensor } \\ \varphi & = & \text { primary variable } \\ \omega & & \text { angular frequency } \\ & & \end{array}$

\section{Introduction}

S OUND generation from a vibrating circular piston is a classical acoustic problem. Significant studies related to $S$ the acoustic field generated by pistons are done by: Freedmann ${ }^{1}$, Lele and Hamilton ${ }^{3}$, Cleveland, et $a l^{4}$, Williams $^{5}$, Blackstock ${ }^{6}$, Cheong and Lee ${ }^{7}$.

The radiation resulting from the baffled piston represents a diverse area of investigation. Some area of researches revolved sound radiation from a boxed loudspeaker or a singing voice, the sound reproduction by electrostatic devices, and the ultrasonic imaging system evaluation.

In this paper, we will compute the sound radiation produced by oscillating baffled pistons using both linear and nonlinear models. The linear wave equation (linear Euler equations) will be employed in the linear analysis, and the Navier-Stokes equations for the nonlinear case. The linear solution will be compared to the Rayleigh integral approximation $^{6,8}$. The nonlinear solution will be compared against linear model for low velocity (less than 0.01 of sound speed). The analytic solutions are used in order to validate the numerical approach. The results will be presented and compared for low and high frequencies.

In practical applications, not only waves radiated from an isolated baffled piston, but also the subsequent development such as interaction between waves from an array of baffled pistons, and radiated wave around complex geometries are of interest. In these circumstances where interference of sound wave occurs, however, the exact solution does not exist, and a numerical approach is needed.

In this paper we will study the nonlinear wave radiation from baffled pistons and their interaction with complex geometry or the interaction from multiple pistons located at different points in 2D space. The challenge of this computation is to capture the behavior of the waves resulting from the interference of the initial waves from the source pistons with other solid objects or other waves. The new waves possess different properties in terms of frequency, amplitude and wavenumber. These parameters are influenced by the initial frequencies and spatial coordinates of the location or complex geometry. The governing equations in Cartesian coordinates are solved using the Optimized Prefactored Compact finite volume (OPC-fv) scheme for spatial discretization by Popescut $e t a l^{9}$. The Low-Dispersion Low-Dissipation Runge-Kutta (LDDRK) by Hu et al ${ }^{10}$ will be used for time discretization. For the treatment of boundary condition, we propose a modified perfect matched layer (PML) which fits better to Navier-Stokes equations. The PML is finished with outflow boundary conditions proposed by Tam and Webb ${ }^{11}$. In order to handle the geometric complexity and the varied wave characteristics in time and space, we have combined the finite-volume approach with i) a Sharp Interface Method (SIM) with cut-cell approach (Ye et $a l^{13}$ ), (Tai and Shyy ${ }^{14}$ ), and ii) local mesh refinement (Berger and Colella ${ }^{15}$ ). 
The governing equations, numerical scheme and various boundary conditions will be discussed briefly in Section II. In this section, we will also present the cut-cell and local mesh refinement techniques highlighting the main ideas in our approach. In section III we present the results and discussions of the numerical simulation of the baffled piston for linear and nonlinear cases. The last section will offer conclusion and remarks.

\section{Numerical Methods}

\section{A. Discretization in Space - The OPC-fv Scheme}

Consider the simplest one-dimensional wave equation

$$
\frac{\partial u}{\partial t}+c \frac{\partial u}{\partial x}=0
$$

To derive the discretized equation, we employ the grid point cluster shown in Figure 1. We focus on the grid point $i$ and the grid points $i-1$ and $i+1$ are its neighbors. The dashed lines define the control volume, and the letters $e$ and $w$ denote the east and west faces, respectively, of the control volume.

For a better understanding of the OPC-fv form, we will start to present of the original finite difference version of the OPC scheme developed by Ashcroft and Zhang ${ }^{16}$, termed OPC-fd. The optimized prefactored compact scheme in the finite difference approach is obtained by the forward and backward operators $D_{i}^{F}$ and $D_{i}^{B}$ such that

$$
\left(\frac{\partial u}{\partial x}\right)_{i}=\frac{1}{2}\left(D_{i}^{B}+D_{i}^{F}\right)
$$

The generic stencils for the forward and backward derivative operators are given by

$$
\begin{gathered}
\eta_{F} D_{i+1}^{F}+\beta_{F} D_{i}^{F}=\frac{1}{\Delta x}\left[a_{F} u_{i+2}+b_{F} u_{i+1}+c_{F} u_{i}+d_{F} u_{i-1}+e_{F} u_{i-2}\right] \\
\beta_{B} D_{i}^{B}+\eta_{B} D_{i-1}^{B}=\frac{1}{\Delta x}\left[a_{B} u_{i+2}+b_{B} u_{i+1}+c_{B} u_{i}+d_{B} u_{i-1}+e_{B} u_{i-2}\right]
\end{gathered}
$$

The finite volume formulation of optimized prefactored scheme is obtained by taking into account equations that describe the approximation of the first order spatial derivative, Eqs. (3) and (4), and the idea that the general form of the function approximation in points $e$ and $w$ has similar forms (approximation of the function in the center of the cell - see Figure 1). To better understand this, first, we consider the one - dimensional problem and assume a unit thickness in the $y$ and $z$ directions. Thus, we obtain

$$
\int_{e}^{w} \frac{\partial u}{\partial t} d x+c\left((A u)_{e}-(A u)_{w}\right)=0
$$

where $(\mathrm{A} u)_{e}$ and $(\mathrm{A} u)_{w}$ are the fluxes across the east and west faces, respectively.

Hence, the discretized wave equation can be written as

$$
\frac{\partial \bar{u}}{\partial t} \Delta x+c\left((A u)_{e}-(A u)_{w}\right)=0
$$

where $\bar{u}$ is the averaged value of $u$ over a control volume.

Based on the OPC-fd scheme, the value of the function in the center of the face is defined by the relations: 


$$
\left\{\begin{array}{l}
u_{e}=0.5\left(u^{F e}+u^{B e}\right) \\
u_{w}=0.5\left(u^{F w}+u^{B w}\right)
\end{array}\right.
$$

where $u^{F e}, u^{B e}, u^{F w}$ and $u^{B w}$ are determined from

$$
\begin{aligned}
& \eta u_{i+1}^{F e}+\beta u_{i}^{F e}=b u_{i+1}-d u_{i} \\
& \eta u_{i+1}^{F w}+\beta u_{i}^{F w}=b u_{i}-d u_{i-1} \\
& \beta u_{i}^{B e}+\eta u_{i-1}^{B e}=b u_{i}-d u_{i+1} \\
& \beta u_{i}^{B w}+\eta u_{i-1}^{B w}=b u_{i-1}-d u_{i}
\end{aligned}
$$

The coefficients in Eq. (8) are the same as those in the OPC-fd scheme (Ascroft and Zhang ${ }^{16}$ ):

$$
\left\{\begin{array}{l}
\eta=\eta_{F}=\eta_{B} \\
\beta=\beta_{F}=\beta_{B} \\
b=b_{F}=-d_{B} \\
d=d_{F}=-b_{B}
\end{array}\right.
$$

B. Time Discretization - Low Dispersion and Dissipation Runge-Kutta (LDDRK) Method

$\mathrm{Hu}$, et $a l^{10}$ considered time integration using the Runge-Kutta algorithm of the differential equation

$$
\frac{\partial u}{\partial t}=F(u)
$$

where the operator $F$ is function of $u$. An explicit $p$-stage algorithm advances the solution of Eq.(10) from the $n^{\text {th }}$ to the $(n+1)^{\text {th }}$ iteration, such that

$$
\begin{aligned}
& u^{(0)}=u^{n} \\
& K^{(1)}=\Delta t F\left(u^{(0)}\right) \\
& \ldots \\
& K^{(i)}=\Delta t F\left(u^{(i-1)}\right) \\
& u^{(i)}=u^{n}+b_{i} K^{(i)} \quad i=1, \ldots, p \\
& \ldots \\
& u^{n+1}=u^{(p)}
\end{aligned}
$$

where $b_{p}=1$.

The coefficients of the Low-Dispersion and Low-Dissipation Runge-Kutta (LDDRK) scheme are obtained such that:

1) the scheme has a certain order of accuracy;

2) the error of the amplification factor of the scheme over the specified phase range is minimized;

3) the amplification factor is within one of the given stability limit.

In this work we use a two-step alternating scheme. The odd step consists of four stages, and the even step has six stages. The scheme presents fourth-order accuracy in time for a linear problem, and second-order accuracy for a nonlinear problem.

The specific procedure is given below.

Odd step (four-stage): 


$$
\begin{aligned}
& K^{(1)}=\Delta t F\left(u^{n}\right) \\
& K^{(2)}=\Delta t F\left(u^{n}+\frac{1}{4} K^{(1)}\right) \\
& K^{(3)}=\Delta t F\left(u^{n}+\frac{1}{3} K^{(2)}\right) \\
& K^{(4)}=\Delta t F\left(u^{n}+\frac{1}{2} K^{(3)}\right) \\
& u^{n+1}=u^{n}+K^{(4)}
\end{aligned}
$$

Even step (six-stage):

$$
\begin{aligned}
& K^{(1)}=\Delta t F\left(u^{n}\right) \\
& K^{(2)}=\Delta t F\left(u^{n}+0.17667 K^{(1)}\right) \\
& K^{(3)}=\Delta t F\left(u^{n}+0.38904 K^{(2)}\right) \\
& K^{(4)}=\Delta t F\left(u^{n}+\frac{1}{4} K^{(3)}\right) \\
& K^{(5)}=\Delta t F\left(u^{n}+\frac{1}{3} K^{(4)}\right) \\
& K^{(6)}=\Delta t F\left(u^{n}+\frac{1}{2} K^{(5)}\right) \\
& u^{n+1}=u^{n}+K^{(6)}
\end{aligned}
$$

In this study, the LDDRK scheme and the OPC-fv scheme are combined. In the context of the above derivation,

$$
F_{l}=-c\left(u_{l}^{e}-u_{l}{ }^{w}\right) / \Delta x,
$$

where $\mathrm{u}_{1}{ }^{\mathrm{e}}=0.5\left(\mathrm{u}_{1}{ }^{\mathrm{Be}}+\mathrm{u}_{1}^{\mathrm{Fe}}\right)$, and $\mathrm{u}_{1}^{\mathrm{w}}=0.5\left(\mathrm{u}_{1}^{\mathrm{Bw}}+\mathrm{u}_{1}{ }^{\mathrm{Fw}}\right)$.

\section{Boundary Treatment}

To handle the outflow boundary for nonlinear case, the perfect matched layer (PML) is adopted. The PML was designed for Euler equation by $\mathrm{Hu}^{18}{ }^{19}$. In order to apply PML to the Navier-Stokes equations, we add the diffusive term into the original PML equations. Finally, the equations that characterize PML are: 


$$
\begin{aligned}
& \frac{\partial u_{1}^{x}}{\partial t}+\sigma_{x} u_{1}^{x}=-\frac{1}{\rho} \frac{\partial p}{\partial x}-u^{x} \frac{\partial u^{x}}{\partial x}+\frac{1}{\operatorname{Re}} \frac{\partial^{2} u^{x}}{\partial x^{2}} \\
& \frac{\partial u_{2}^{x}}{\partial t}+\sigma_{y} u_{2}^{x}=-u^{y} \frac{\partial u^{x}}{\partial y}+\frac{1}{\operatorname{Re}} \frac{\partial^{2} u^{x}}{\partial y^{2}} \\
& \frac{\partial u_{1}^{y}}{\partial t}+\sigma_{x} u_{1}^{y}=-u^{x} \frac{\partial u^{v}}{\partial x}+\frac{1}{\operatorname{Re}} \frac{\partial^{2} u^{y}}{\partial x^{2}} \\
& \frac{\partial u_{2}^{y}}{\partial t}+\sigma_{y} u_{2}^{y}=-\frac{1}{\rho} \frac{\partial p}{\partial y}-u^{y} \frac{\partial u^{y}}{\partial y}+\frac{1}{\operatorname{Re}} \frac{\partial^{2} u^{y}}{\partial y^{2}} \\
& \frac{\partial p_{1}}{\partial t}+\sigma_{x} p_{1}=-\gamma p \frac{\partial u^{x}}{\partial x}-u^{x} \frac{\partial p}{\partial x} \\
& \frac{\partial p_{2}}{\partial t}+\sigma_{y} p_{2}=-\gamma p \frac{\partial u^{y}}{\partial y}-u^{y} \frac{\partial p}{\partial y} \\
& \frac{\partial \rho_{1}}{\partial t}+\sigma_{x} \rho_{1}=-\frac{\partial\left(\rho u^{x}\right)}{\partial x} \\
& \frac{\partial \rho_{2}}{\partial t}+\sigma_{y} \rho_{2}=-\frac{\partial\left(\rho u^{y}\right)}{\partial y}
\end{aligned}
$$

Where $u^{x}, u^{y}$ indicate velocity in $x$ and $y$ direction, and $\sigma_{x}$ and $\sigma_{y}$ is defined by the formula:

$$
\sigma=\sigma_{m}\left(\frac{d}{D}\right)^{\beta}
$$

where $\sigma_{m}=$ maximum value of $\sigma$ (the absorbing coefficient for PML domain), $D=$ the width of the PML domain, $d$ $=$ distance from its interface to the interior domain (see Figure 2). The PML is finished with outflow boundary designed by Tam and Webb ${ }^{11}$ :

$$
\frac{\partial p_{i}}{\partial t}+\cos (\theta) \frac{\partial p_{i}}{\partial x}+\sin (\theta) \frac{\partial p_{i}}{\partial y}+\frac{p_{i}}{2 R}=0
$$

where $i=1$, 2. In our computation, we will use 21 points in PML. The results are satisfactory with this kind of approach of boundary condition (the reflection will be negligible even the waves have high amplitude, or in the domain where the wave are powerful dissipated).

\section{Cut-Cell Approach for Complex Geometry}

Cut-cell approach is a Cartesian grid method that is used to handle the complex geometry in sound filed. The background grid is a Cartesian grid and the explicit interfaces are used to divide entire domain to different phases/regions. The interfacial cells will be cut to fit better the interface. The boundary condition will be applied at the interface/ cut-sides for each phases/regions. Each phase/region will be calculated separately and connected by the boundary condition at the interface.

In the cut-cell approach, there are three main steps, namely, (i) interface/boundary identification, (ii) construction of the data structures among the boundary cells, and (iii) the flux computations in the boundary region. In this work, the first and second steps are further developed. The third step, the flux computation near the boundary, follows that originally reported by Popescu $e t a l^{20}$.

In the first step, the geometric definition of the solid boundary along with the intersections between the boundary and the neighboring grid must be established. These intersections will be the cut-points and used for the next step, the absorption step. In the second step, because of the interface, some cells are cut and can not maintain the rectangular shape anymore. These cut-cells have to be treated specially. An absorption technique is applied here. The fragments of cells can be absorbed by neighboring cells or larger fragments to form cut-cells. That means the cells around the interface have to be reconstructed. For the third step, a so called 25-point method is used to handle the fluxes near the interface. 
A test case with an oblique ellipse will be discussed in section III. Figure 3 is an example of cut-cells based on different grid sizes. It is clear that once the grid size decreases, the more smooth geometry can be obtained.

\section{E. Local Grid Refinement for OPC-FV Scheme}

In order to support the requirement for employing the OPC-fv scheme, an overlapping-block-type refinement technique is developed. This local grid refinement technique can be illustrated by Figure 4 . The coarse grid represents the original grid system and the fine grid is based on the local grid refinement. In the OPC scheme, the cells E, F and G are used to calculate the information at the right boundary of the coarse grid. Therefore, the value of the primary variables must be accurate at the cell centers of E, F and G. Specifically, the values at E, F and G are obtained from the fine grid. Similarly, the information needed by fine cells 1, 2 and 3 is obtained from the coarse grid (original grid). Using this kind of algorithm, the fluxes can be transferred between two blocks to facilitate the OPC scheme.

In the present grid refinement technique, in order to maintain the conservative property and to reduce the error, the following procedure is adopted (based on the data structure shown in Figure.5):

1. Primary variables on coarse grid $\left(\Phi_{2}\right)$, employing the finite volume approach:

$$
\Phi_{2} \Delta V_{\Phi_{2}}=\phi_{3} \Delta V_{\phi_{3}}+\phi_{4} \Delta V_{\phi_{4}}
$$

2. Primary variables on fine grid $\left(\phi_{1}, \phi_{2}\right)$, employing an high order interpolation method to find $\phi_{1}^{*}$ and $\phi_{2}^{*}$. To reduce the error from the interpolation, the error between corresponding fine and coarse grids, computed as, $\varepsilon=\Phi_{1} \Delta V_{\Phi_{1}}-\phi_{1}^{*} \Delta V_{\phi_{1}}+\phi_{2}^{*} \Delta V_{\phi_{2}}$, is used for adjusting the value of fine grid to improve the conservative property between two grids. At the end, the primary variables in fine grid are modified as:

$$
\left\{\begin{array}{l}
\phi_{1}=\phi_{1}^{*}+\varepsilon / 2 \\
\phi_{2}=\phi_{2}^{*}+\varepsilon / 2
\end{array}\right.
$$

\section{Results and Discussion}

The origin of the coordinate system will be chosen in the center of the piston, as shown in Figure 6. We use the following dimensionless variables with respect to the following scales:

- Length scale $=$ diameter of piston, $2 a$

- Velocity scale $=$ speed of sound, $c$

- Time scale $=2 a / c$

- Density scale $=$ undisturbed density, $\rho_{0}$

- Pressure scale $=\rho_{0} c^{2}$

- Frequency $=c / 2 a$

\section{A. Two dimensional Linear Baffled Piston}

The linear Euler equations are:

$$
\left\{\begin{array}{l}
\frac{\partial \vec{u}}{\partial t}+\nabla p=0 \\
\frac{\partial p}{\partial t}+\nabla \cdot \vec{u}=0
\end{array}\right.
$$

The initial conditions are set as follows: 


$$
\left\{\begin{array}{l}
u^{x}(x, y)=0 \\
u^{y}(x, y)=0 \\
p(x, y)=0
\end{array}\right.
$$

For this problem, two boundary conditions are used, at the wall, and in the far field. The boundary conditions at the wall with the piston are:

$$
u^{y}(x, 0)=\left\{\begin{array}{cl}
V_{0} \cos (\omega t) & (x, 0) \in \text { piston } \\
0 & \text { otherwise }
\end{array}\right.
$$

where $\omega$ is the angular frequency of the piston, and $V_{0}$ is the amplitude of the displacement. For small signals, the solution can be obtained using the linear equation. Therefore, the value of $V_{0}$ influences only the amplitude of the solution, and none of the other wave parameters. For this reason we can make $V_{0}$ to equal to 1 .

The outflow boundary condition is based on the acoustic radiation condition of Tam and Webb ${ }^{11}$ :

$$
\left\{\begin{array}{l}
\frac{\partial \vec{u}}{\partial t}+\nabla p=0 . \\
\frac{\partial p}{\partial t}+\frac{\partial p}{\partial x} \cos \theta+\frac{\partial p}{\partial y} \sin \theta+\frac{p}{2 R}=0 .
\end{array}\right.
$$

where $R$ is distance from origin of the $\mathrm{x}$ axis (center of the piston) to the point $(x, y)$. The analytical solution is given by the Rayleigh integral ${ }^{6,8}$

The piston behavior is presented in terms of the Helmholtz number, $k a$, where $k$ is wavenumber, and $a$ is the radius of the piston

$$
k a=\frac{\omega a}{c}
$$

Next, we take a closer look at the wave behavior by studying the directivity factor for two frequencies: low frequency $(k a=2)$ and high frequency $(k a=8)$. The directional characteristic of a source is described by the amplitude directivity factor $D$, defined as the pressure at any angle to the pressure on the angle of maximum pressure.

$$
D(\theta)=\frac{p(R, \theta)}{p(R, \pi / 2)}
$$

where the pressure is computed at any arbitrary time $t$. It is clear that the radiation will be strongest on the $y$-axis. This is why we took maximum pressure at $\theta=\pi / 2$. The directivity factor indicates how effectively that a directional source concentrates its available acoustic power into a preferred direction.

In case that $k a$ is low, the piston size is small compared with the wavelength of the sound and in this case the behavior of a piston is like a point source; hence, the directivity pattern does not have a preferred direction. When $k a$ » 1 , the directive function has nulls, and between the nulls are secondary radiation maximum, of monotonically decreasing prominence. The number of nulls and secondary maximum is determined by the size of $k a$, where $a$ is the radius of piston, and $k a=2 \pi a / \lambda$. In other words, the number of the lobes increases with the value of $k a$. .In our calculation, we compare the analytical and numerical values of beam pattern for $k a=2$ and $k a=8$. As shown in Figure 7, the numerical solution compares favorably with the analytical beam pattern.

\section{B. Two - Dimensional Nonlinear Baffled Piston}

The governing equations are: 


$$
\begin{aligned}
& \frac{\partial \rho}{\partial t}+\frac{\partial\left(\rho u_{j}\right)}{\partial x_{j}}=0 \\
& \frac{\partial\left(\rho u_{i}\right)}{\partial t}+\frac{\partial\left(\rho u_{i} u_{j}\right)}{\partial x_{j}}+\frac{\partial p}{\partial x_{i}}=\frac{\partial \tau_{i j}}{\partial x_{j}} \\
& \frac{\partial p}{\partial t}+u_{j} \frac{\partial p}{\partial x_{j}}+\gamma \frac{\partial u_{j}}{\partial x_{j}}=0
\end{aligned}
$$

where

$$
\tau_{i j}=\frac{1}{R_{D}}\left(\frac{\partial u_{i}}{\partial x_{j}}+\frac{\partial u_{j}}{\partial x_{i}}\right)
$$

$R_{D}=2 a c / v$ is the Reynolds number based on diameter of the piston, $v$ is the kinematic viscosity of the fluid, and $\gamma$ is the ratio of specific heat.

The initial conditions are:

$$
\left\{\begin{array}{l}
\rho=1 . \\
p=1 / \gamma \\
u=0 . \\
v=0
\end{array}\right.
$$

The boundary conditions at the wall with the piston are

$$
v(x, 0, t)= \begin{cases}V_{0} \cos (\omega t) & (x, 0) \in \text { piston } \\ 0 & \text { otherwise }\end{cases}
$$

For outflow boundary, we use perfect matched layer (PML) that is finished with outflow boundary condition designed by Tam and Webb ${ }^{11}$.

We will present the results for two situations: low frequency $(k a=2)$ and high frequency $(k a=8)$.

\section{Low frequency $(k a=2)$}

The computation is done in a domain $(x, y) \in[-5,5] \times[0,10]$ and a grid $161 \times 161$. The time step is based on CFL $=$ 0.2 , where CFL $=c \frac{\Delta t}{\Delta x}$.

First we present a solution for which $V_{0}$ is small. In this case, the linear model can approximate the solution. The solution and contour plot for $V_{0}=0.01 \mathrm{c}$ are presented in the Figure 8 (a) and (b). We can see that the solution is very close to the linear approach. This is expected because we have the case of a small signal, when the solution can be approximated very well with the linear behavior.

Blackstock ${ }^{6}$ shows that in the plane progressive waves, the speed of sound can be approximated by $c+\beta u$, where $\beta$ is the coefficient of nonlinearity. Not all points on the wave move at the same speed. At the point with $u=0$, the wave essentially moves at the speed of sound $c$. However, at the peak, where $u$ has its greatest value due to nonlinear effects, the wave travels the fastest. At the trough, where $u$ is the lowest, the wave is slowest. Consequently, the peak tends to catch up with the trough, as shown in sketches (a), (b), and (c) in Figure 9. The multi-valued waveform is physically impossible. The effects of viscosity and heat conduction prevent actual multivalueness. Dissipation becomes very important whenever any segment of the waveform becomes very steep. Very steep segments are actually shock waves.

The computational results show that the solution becomes steeper when $V_{0}$ increases, and the dissipation become very important because speed of sound is bigger $(c+\beta u)$. This is illustrated in Figure 10 (a), (b) and (c), where the linear solution is plotted to emphasize that dissipation increases as the initial amplitude grows. In Figure 10(d), we 
present the contour plot of a dissipative solution. We can see that boundary condition assures again a negligible reflection from the boundary.

2. High frequency $(k a=8)$

The computation is done by a domain $(x, y) \in[-2.5,2.5] \times[0,5]$ and a grid $251 \times 251$. The time step is based on $\mathrm{CFL}=0.2$, where $\mathrm{CFL}=c \frac{\Delta t}{\Delta x}$. In this case, we use a finer grid to be able to capture the behavior of high frequency. As shown in Figure 11, for the case of weak signals $\left(V_{0}=0.01\right)$, the solution is well approximated with a linear behavior, except that the nonlinear computation captures the detailed behavior around the piston, namely, the diffraction because of the discontinuity around of the edge of the piston (see Figure 11(b) and (c)). For the case of a high frequency wave radiated from a baffled piston dissipates faster than in the case of the low frequency. This happens because the sped of a high frequency wave is larger, hence speed of sound $(c+\beta u)$ is higher, and the diffusion becomes more powerful. This phenomenon is observed in our computation (see Figure 12(a)). Again we notice that actual boundary condition yields a negligible reflection also in this case (see Figure 12(b)).

\section{Array of Baffled Piston}

The problem consists of solving the radiation interference from more than one piston. This is shown in Figure 13, where three pistons are considered. The challenge of the numerical solution will consist of capturing the behavior of the new waves, particularly the range of wavenumbers.

The proposed approach is solved using

- the Navier Stokes equations

- wall boundary conditions and piston source characteristics for wall-embedded pistons - Eq.(15)(16)

- $\quad$ outflow boundary conditions based on PML and acoustic radiation conditions of Tam and Webb ${ }^{11}$ , Eq. (17)

Next we study the interference of the waves of three source when the source is characterized by low frequency $(k a=2)$ and high frequency $(k a=8)$.

\section{Low frequency $(k a=2)$}

In case of the low frequency, the source is characterized by the directivity pattern without nulls and maxims; in other words, the radiated waves do not have a preferred direction. In case of a weak signal $\left(V_{0}=0.01\right)$ the interference wave will have direction that follow the radial direction to the sources (see Figure 14 (a) and (b)). In case of the strong signal, the waves quickly become very steep and with high amplitudes near the source, and dissipate quickly. The consequence of this behavior is that even when the radiated waves interfere, we recognize that the pattern of the initial source in the zone that is close to the sources - where the amplitude of wave is not negligible. The maxima and minima follow the directivity pattern of the sources. This behavior is easily recognized in our computation (see Figure 14 (c) and (d)).

\section{High frequency $(k a=8)$}

A high frequency source is characterized by an efficiently radiated wave in the direction of the axis of the piston. In this case the interference between radiated increases in the same time with decreasing of the distance between the centers of pistons. When the pistons are close enough, the interference waves are very well defined, and their position and power depend on the position of the source. This behavior is captured very well by our computation so we can see in Figure 15.

In the case that the piston emits a strong signal, the shock wave appears very close to the piston and waves dissipated very fast; hence, the amplitude decays very fast, which can be seen in the previous computation. In this case an important interference between waves can be seen only very close to the pistons, and a very low amplitude interference wave is far away of the piston (see Figure 16).

\section{Two - Dimensional Linear Baffled Piston with an Elliptical Solid Object}

The purpose of this case is to test the ability of cut-cell method. The computational domain (sound field) is a square with domain $(x, y) \in[-5,5] \times[0,10]$ and a baffled piston with radius 0.5 is mounted at the bottom side of the domain. In this piston problem, the small signal condition is assumed and the linear Euler equations are used. The governing equation, the initial condition and the periodical velocity at the surface of piston are same as linear baffled piston case from previous sections. The CFL is 0.5 in this case.

- A small solid ellipse is placed at the center of this domain for which 
- the long axis is 0.5

- $\quad$ the short axis is 0.25

- The origin of ellipse is situated in $(0 ., 0$.) point

- The ellipse is inclined at the 30-degree angle.

In this section, a grid refinement test is used to assess the convergence of the solution, which Figure 17 shows the geometry and the pressure distribution at $x=0$ along the $\mathrm{y}$ axis at $t=10$ based on different grid resolutions. As the grid is refined, the solutions converge toward each other, demonstrating that the cut-cell approach works satisfactorily in regard to yielding unique solutions.

\section{E. Two - Dimensional Linear Baffled Piston with Local Grid Refinement}

To consider a baffled piston problem with the geometry $10 \times 10, k a=4$, the initial and boundary condition are the same as that in previous section. In this case, the high variation zone is around $(x, y) \in[-2,2] \times[0,5]$ and it is selected for grid refinement.

The detailed geometry of this test case is shown in Figure 17. The geometry of the entire domain is $10 \times 10$, $(x, y) \in[-5,5] \times[0,10]$ and a refined domain is $4 \times 5,(x, y) \in[-2,2] \times[0,5]$. Inside the refined domain, the grid point in both $\mathrm{x}$ and $\mathrm{y}$ directions are doubled. In the following discussion, the refined cases will be denoted "(refined)".

Once the refined area is chosen, the entire domain is divided into the regular and refined domain. Figure 17 is the data structure between regular and refined grid. An inner computational boundary of coarse grid has to be set first. The function of this boundary zone is to transfer the information from the fine grid to coarse grid. The coarse cells outside the new domain will not be calculated during the computation. Because the computational domain is not rectangular, the computational chains of OPC-FV schemes on coarse grid have to be redefined. The solid lines in different color (black and red) with the arrows in Figure 17 are the demonstrations of the new computational chains of the OPC-FV scheme in each domain. Once this information is ready, coarse grid and fine grid can be calculated separately and the information can be transferred to each other by the inner computational boundary of coarse grid and outer computational boundary of fine grid.

In this test case, three different grids are selected for testing: 100×100(refined), 200×200(refined) and $300 \times 300$ (refined) and the results of the cases without grid refinement technique will be used for comparison.

Table 1 is the summary of usage of grid points and CPU time of all cases and the Figure 18 shows the error of each case. Here, the cases with $100 \times 100,200 \times 200$ and $100 \times 100$ (refined) grid are selected for comparison. By Table 1 and Figure 18, it can be seen that the error of $100 \times 100$ (refined) is close to the case $200 \times 200$ and better than $100 \times 100$. For comparison of the CPU time and usage of memory, the case $100 \times 100$ (refined) only uses $33 \%$ CPU time and $45 \%$ memory and achieve similar resolution of the case with $200 \times 200$ grid. The error of the cases with equivalent grid points is also compared. For the case with $100 \times 100$ (refined) grid, its equivalent case is $135 \times 135$. By Table 1 and Figure 22, the error of $100 \times 100$ (refined) is less than its equivalent case but extra $14 \%$ CPU time is used. Another case is $200 \times 200$ (refined) and it equivalent case, $269 \times 269$, the error of former is less and extra $11 \%$ CPU time is used. By these comparisons, it can illustrate that the current refinement technique can save the CPU time and the usage of memory and improve resolution of results.

\section{Concluding Remarks}

Numerical computations of the baffled piston have been conducted with both linear and nonlinear models. For nonlinear waves, depending on the flow parameters, the propagation, interference, sharpening, and dissipation of the wave characteristics are well captured. For example, for the weak signal case, since the dissipation is less important, we observe the pattern of the interfering waves. For a stronger signal, the shock wave appears close to the pistons, and experiences dissipation quickly. To handle the complex geometry, the performance of the cut-cell approach is assessed. By combining the cut-cell and local grid refinement techniques, both geometric flexibility and adaptively refined resolutions can be attained.

\section{Acknowledgments}

The present work was sponsored in part by NASA and the US Air Force References 


\section{References}

${ }^{1}$ Freedman, A., “Acoustic Field of A Pulsed Circular Piston”, J. Sound Vib., Vol.170, No.4, 1994, pp.459-519

${ }^{2}$ Lele ,Y.S and Hamilton, M.F., “ Time-domain modeling of pulsed finite-amplitude sound beams”, J. Acoust. Soc. Am, Vol.97, No.2, 1995, pp.906-917

${ }^{3}$ Cleveland, R.O., Hamilton, M.F. and Blackstock, D.T.,“ Time-domain modeling of finite-amplitude sound in relaxing fluids”, J. Acoust. Soc. Am, Vol.99, No.6, 1996, pp.3312-3318

${ }^{4}$ Williams, E.G., Fourier Acoustics Sound Radiation and Nearfield Acoustical Holography, Academic Press, Dan Diego, 1999

${ }^{5}$ Blackstock, D.T., Fundamentals of Physical Acoustic, A Wiley-Interscience publication Wiley, New York, 2000

${ }^{6}$ Cheong, C. and Lee, S., "The Effect of Discontinuous Boundary Conditions on the Directivity of Sound from a Piston", $J$. Sound Vib., Vol.239, No.3, 2001, pp.423-443

${ }^{7}$ Williams, E.G., Fourier Acoustics Sound Radiation and Nearfield Acoustical Holography, Academic Press, Dan Diego, 1999

${ }^{8}$ Popescu, M., Shyy, W., and Garbey, M., “A Study of Dispersion-Relation-Preserving and Optimized Prefactored Compact Schemes for Wave Equation”, J. Comput. Phys., Vol.210, No.5, 2005, pp.705-729

${ }^{9}$ Hu, F.Q., Hussaini, M.Y. and Manthey, J.L.,” Low Dissipation and Dispersion Runge-Kutta for Computational Acoustics,” J. Comput. Phys., Vol.124, 1996, pp.177-191

${ }^{10}$ Tam, C.K.W., and Webb, J.C., "Radiation Boundary Condition and Anisotropy Correction for Finite Difference Solutions of the Helmholtz Equation”, J. Comput. Phys., Vol.113, No. 1, 1994, pp.122-133

${ }^{11}$ Tam, C.K.W., Webb, J.C., and Dong, Z., "A study of the Short Wave Components in Computational Acoustics," J. Comput. Acoust., Vol.1, 1993, pp.1-30

${ }^{12}$ Ye, T., Shyy, W., Tai, C., and Chung, J., “Assessment of Sharp- and Continuous-Interface Methods for Drop in Static Equilibrium ” Computers and Fluids, Vol.33, 2004, pp.917-926

${ }^{13}$ Tai, C., and Shyy, W., "Multigrid Computations and Conservation Law Treatment of a Sharp Interface Method", Numerical Heat Transfer: Part B, Vol.48, No.5, 2005, pp.405-424

${ }^{14}$ Berger, M. J., and Colella, P, “Local Adaptive Mesh Refinement for Shock Hydrodynamics”, J. Comput. Phys., Vol.82, 1989, pp.64-84

${ }^{15}$ Ashcroft, G., and Zhang, X., “Optimized Prefactored Compact Scheme”, J. Comput. Phys., Vol.190, 2003, pp.459-477

${ }^{16}$ Hu, F.Q., Hussaini, M.Y. and Manthey, J.L.,” Low Dissipation and Dispersion Runge-Kutta for Computational Acoustics”, J. Comput. Phys., Vol.124, 1996, pp.177-191

${ }^{17}$ Hu, F.Q., “On Perfectly Matched Layer As An Absorbing Boundary Condition”, 1996, AIAA Paper, pp. 96-1664

${ }^{18}$ Hu, F.Q., “A Stable, Perfectly Matched Layer for Linearized Euler Equations in Unsplit Physical Variables” J.Comput. Phys., Vol.173, 2001, pp.255-480

${ }^{19}$ Popescu, M., Tai, C., and Shyy, W. “A Finite Volume-Based High Order Cartesian Cut-Cell Method for Computational Aeroacoustics”, 2005, AIAA Paper, 05-2825

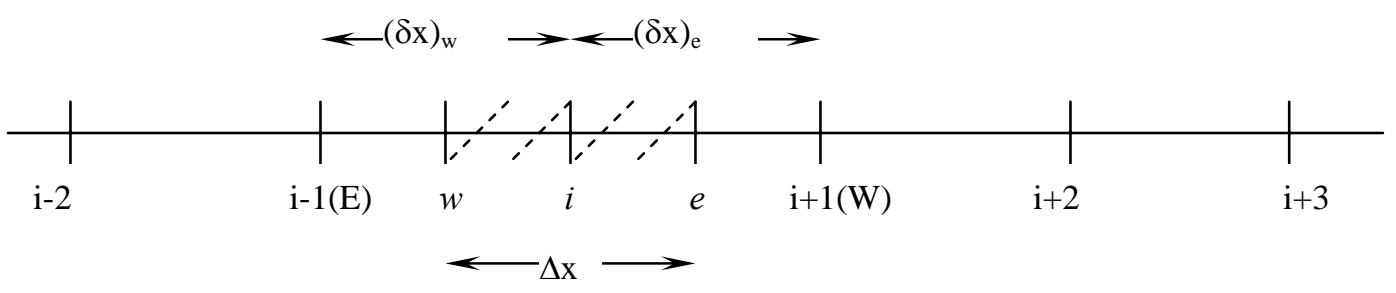

Figure 1.Grid points cluster for one-dimensional problem 


\begin{tabular}{|l|l|l|}
\hline$\sigma_{x} \neq 0$ & $\sigma_{y} \neq 0$ & $\sigma_{x} \neq 0$ \\
$\sigma_{y} \neq 0$ & $\sigma_{x}=0$ & $\sigma_{y} \neq 0$ \\
\hline & & \\
& & $\sigma_{y}=0$ \\
$\sigma_{y}=0$ & & $\sigma_{x} \neq 0$ \\
$\sigma_{x} \neq 0$ & & \\
& & \\
& & \\
\hline
\end{tabular}

Figure 2. Illustration of computational domain with Perfect Matched Layers as outflow boundary

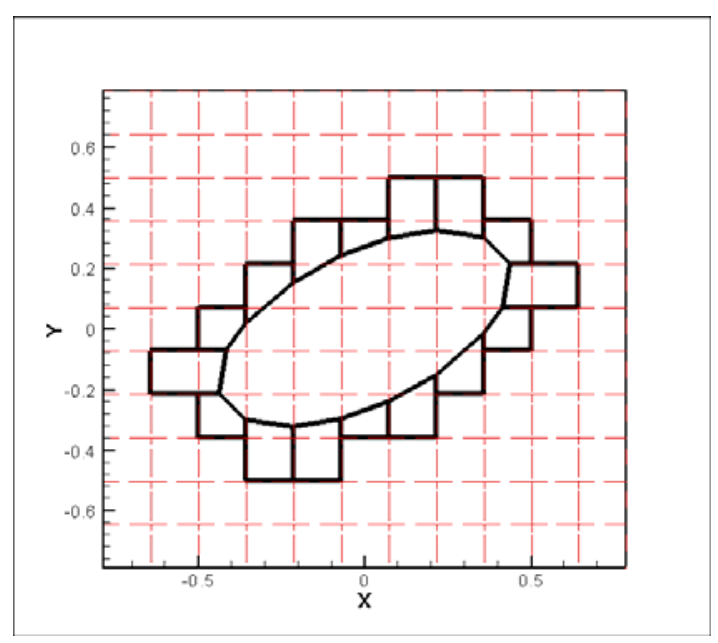

a)

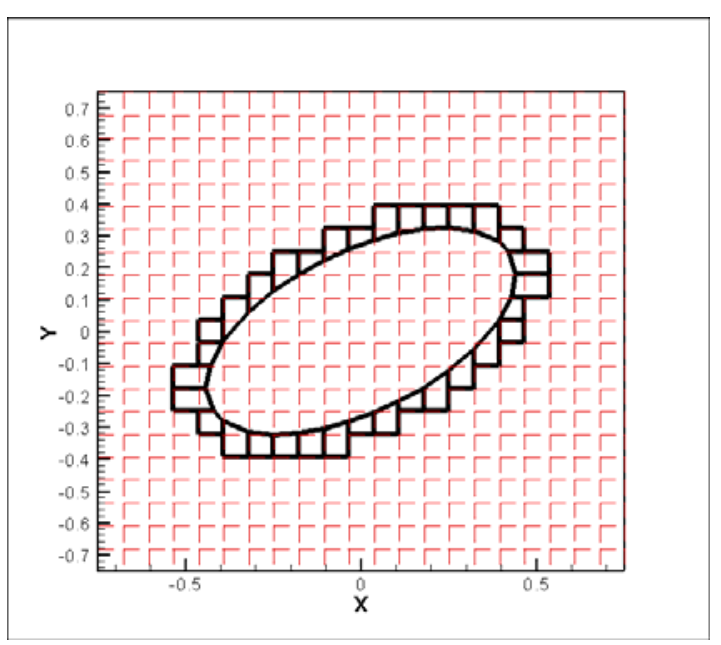

b)

Figure 3. Example of mixed structure and unstructured grid

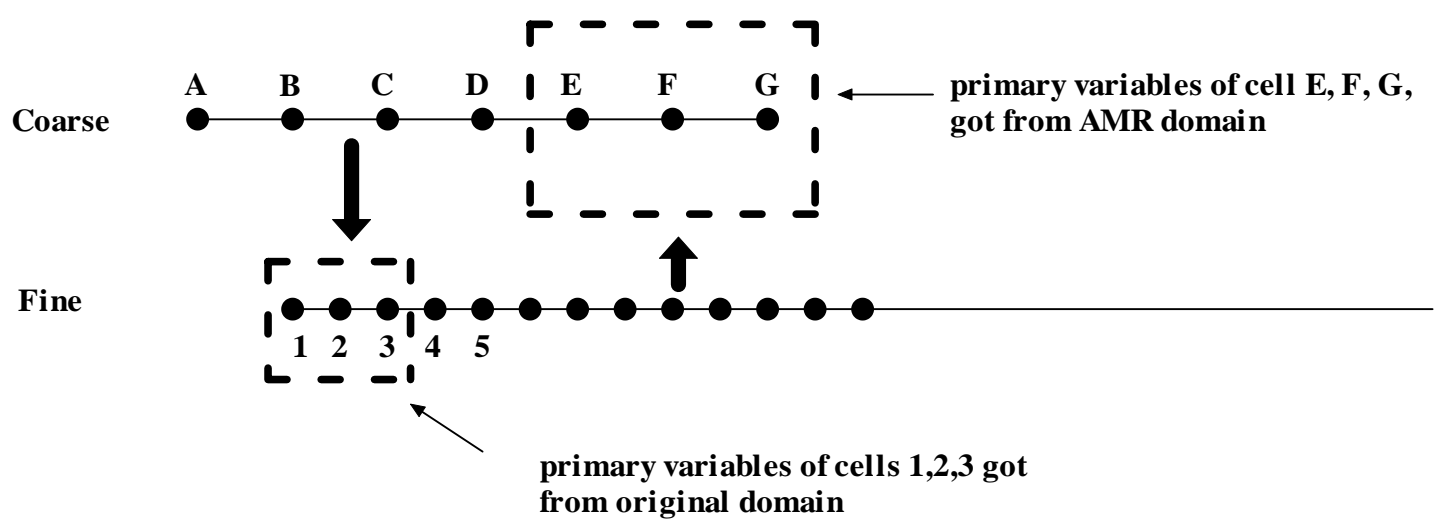

Figure 4. Illustration of the overlapping-block-type grid refinement technique 
Fine grid

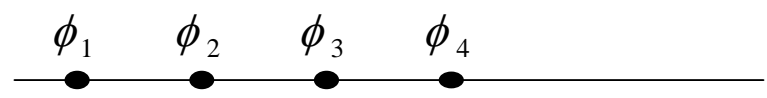

Coarse grid

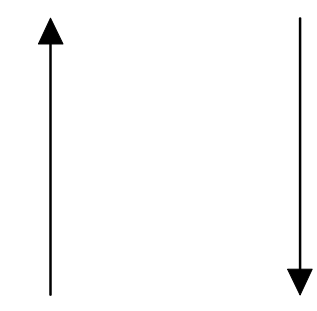

$\Phi_{1} \quad \Phi_{2}$

Figure 5. Illustration of conservative local mesh refinement method

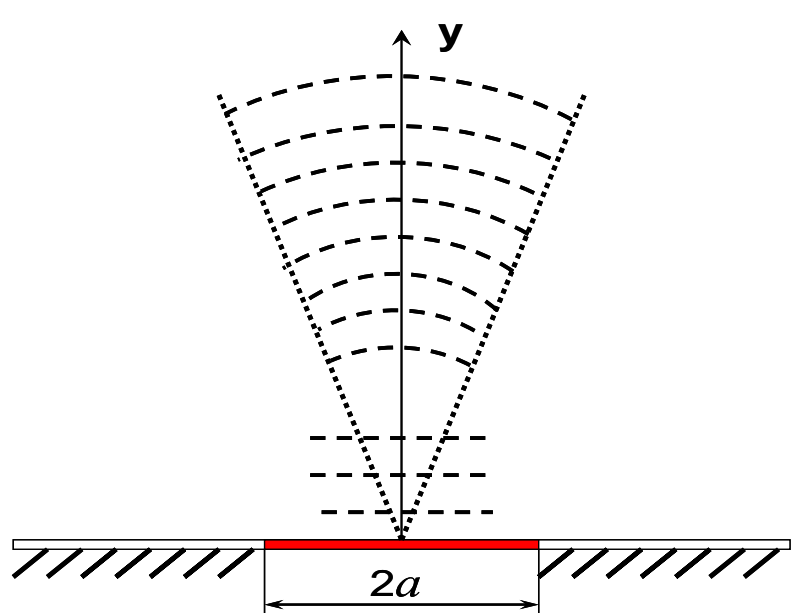

a)

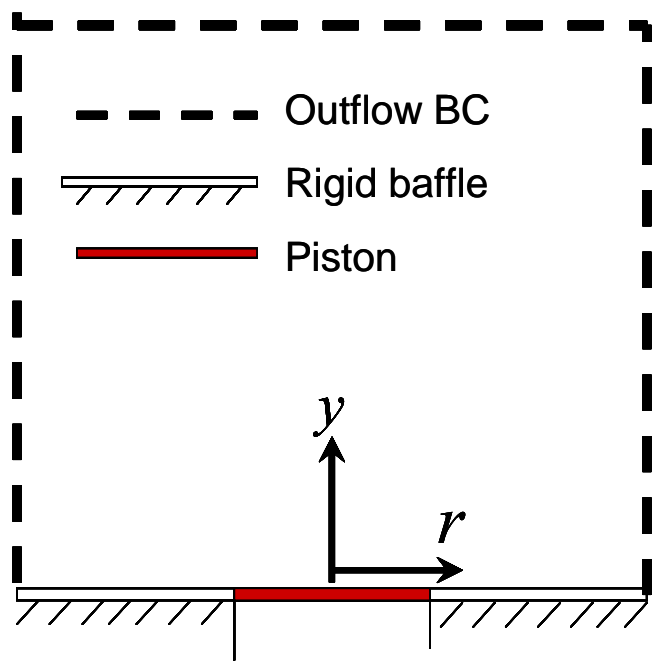

b)

Figure 6. Radiation from a baffled piston : a)general description; b)boundary condition

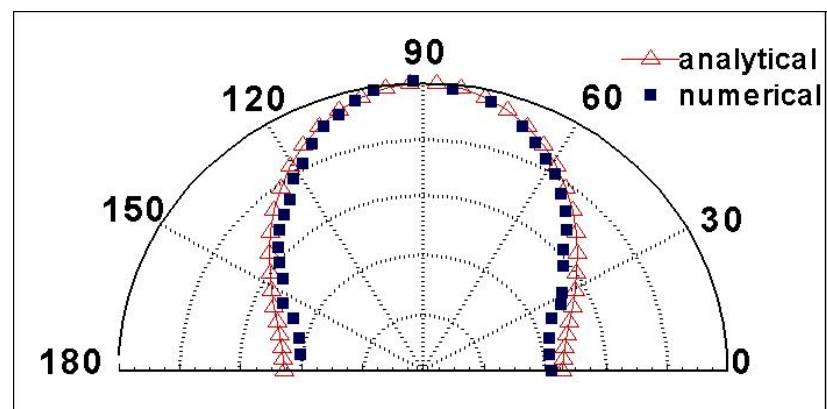

a)

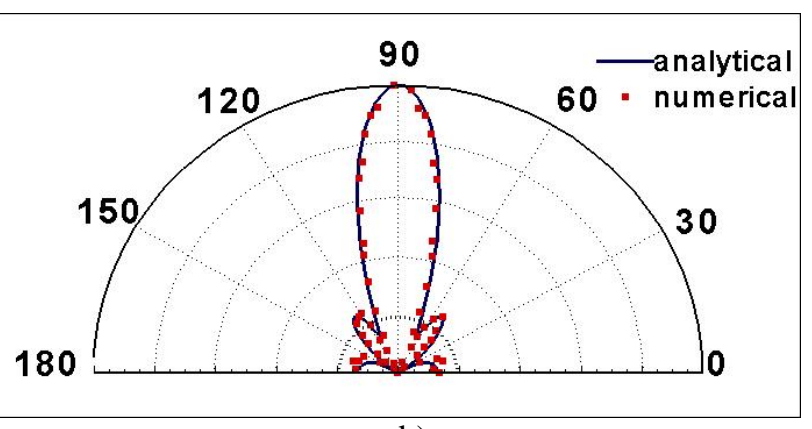

b)

Figure 7. Radiation from a baffled piston: Beam Patterns $|D(\theta)|$ - 2D linear Piston:. a) $k a=2$; b) $k a=8$ 


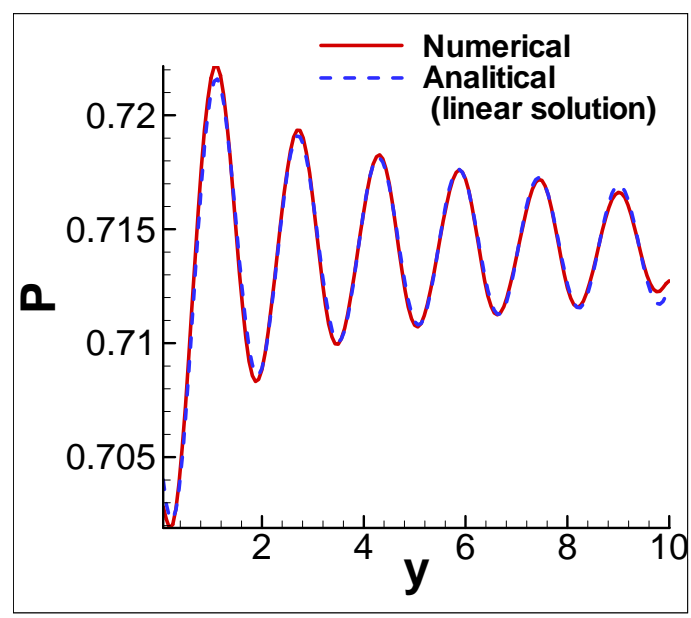

a)

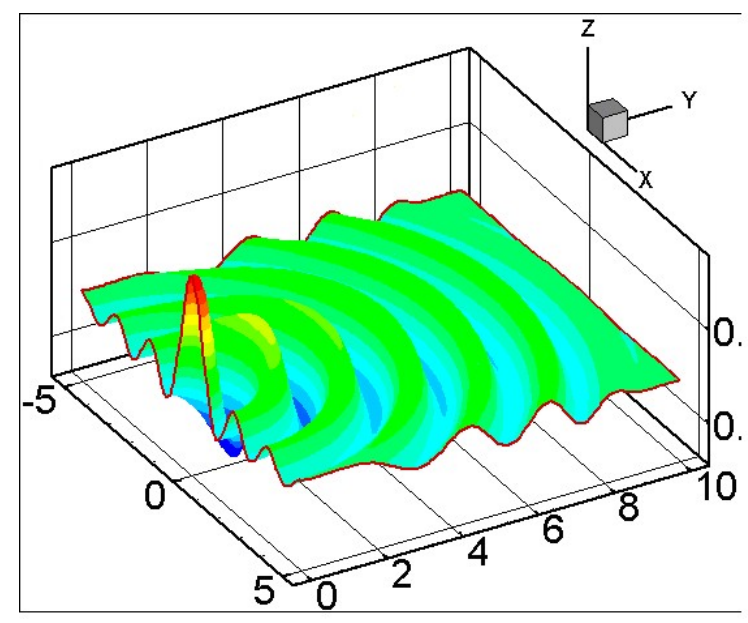

b)

Figure 8. Nonlinear baffled piston: $t=12, \mathrm{CFL}=0.2, V_{0}=0.01 c_{0} ; k a=2:$ a) Axial solution ; b) Contour plot

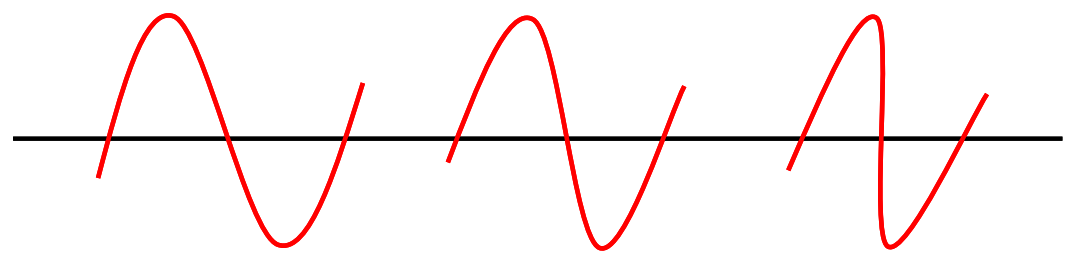

a)

b)

c)

Figure 9. Cumulative distortion of a plane progressive wave

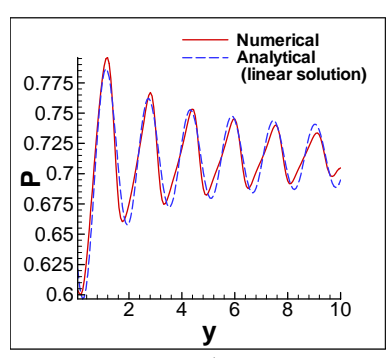

a)

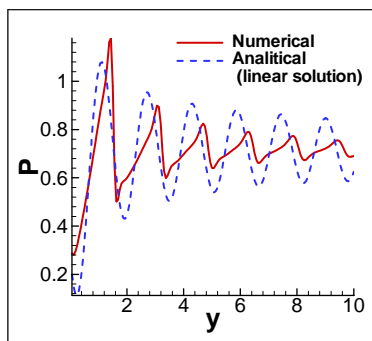

b)

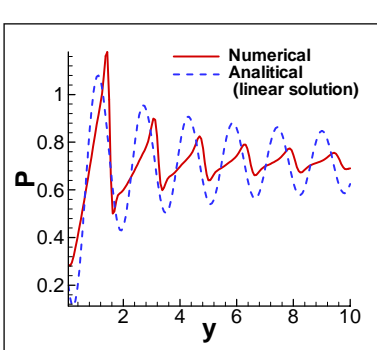

c)

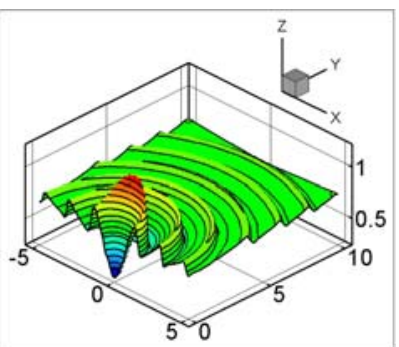

d)

Figure 10. Axial solution: $t=12$; $k a=2 \mathrm{CFL}=0.2$ - Nonlinear baffled piston : a) $V_{0}=0.1 c_{0}$ b) $\left.V_{0}=0.3 c_{0} ; \mathrm{c}\right) V_{0}=$ $0.5 c_{0}$ d) 3D contour plot, $V_{0}=0.5 c_{0}$ 


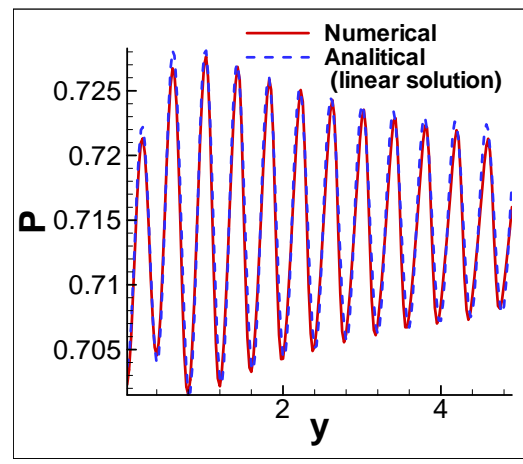

a)

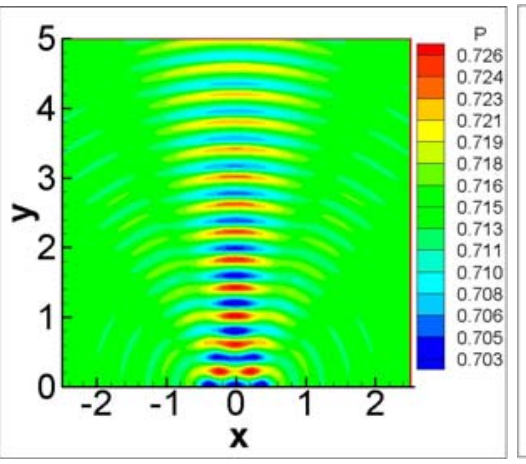

b)

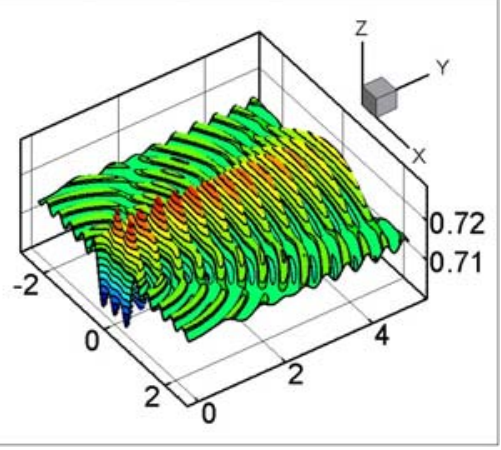

c)

Figure 11. Nonlinear baffled piston: $t=12, \mathrm{CFL}=0.2: V_{0}=0.01 c_{0} ; k a=8$, - a)Axial solution; b) 2D Contour plot; c) 3d Contour plot

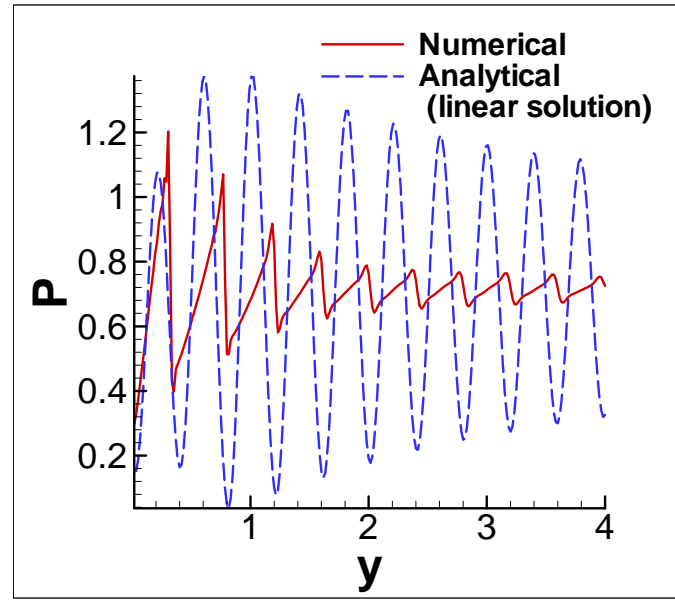

a)

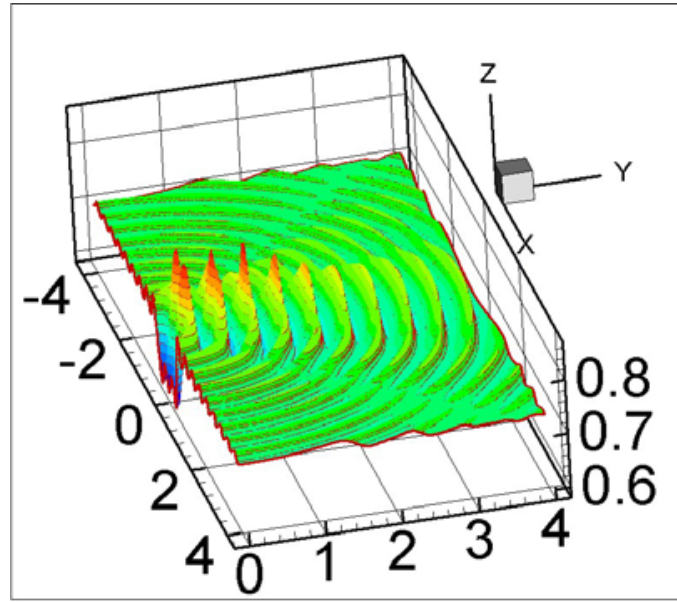

b)

Figure 12. Radiation wave from a baffled piston -: $t=12, V_{0}=0.5 c_{0} ; k a=8$ - Nonlinear baffled piston: a)Axial solution; b) Contour plot

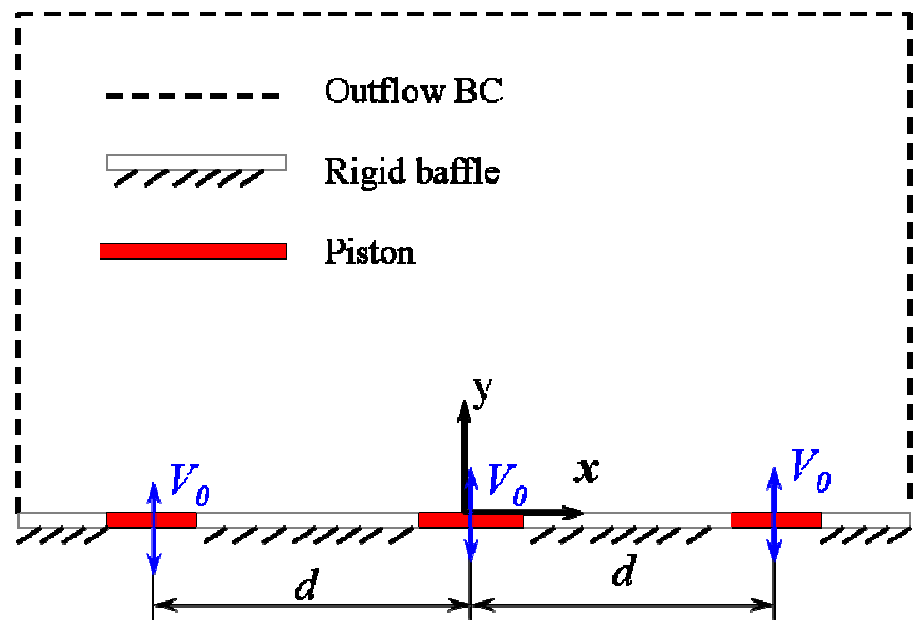

Figure 13. Array of the baffled pistons: General presentation 


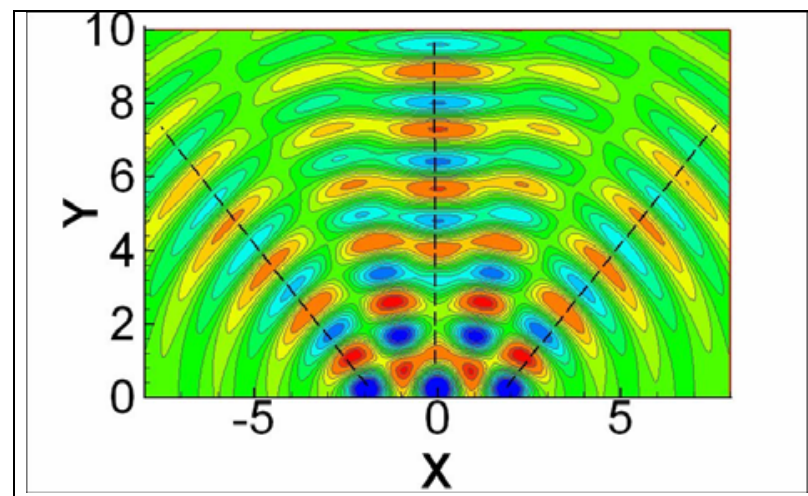

a)

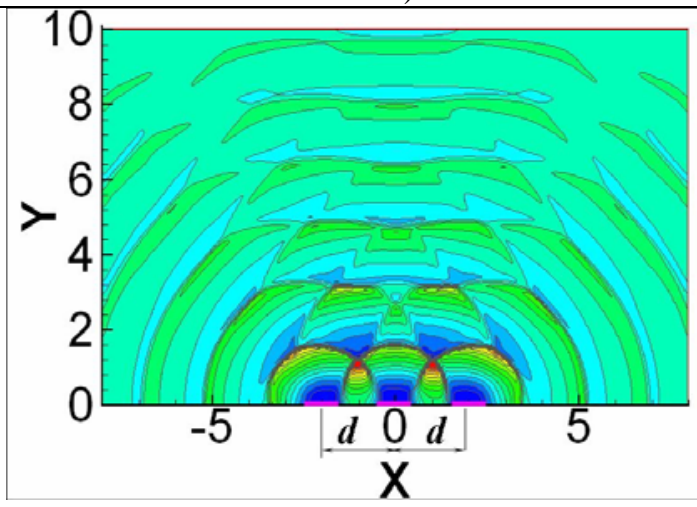

c)

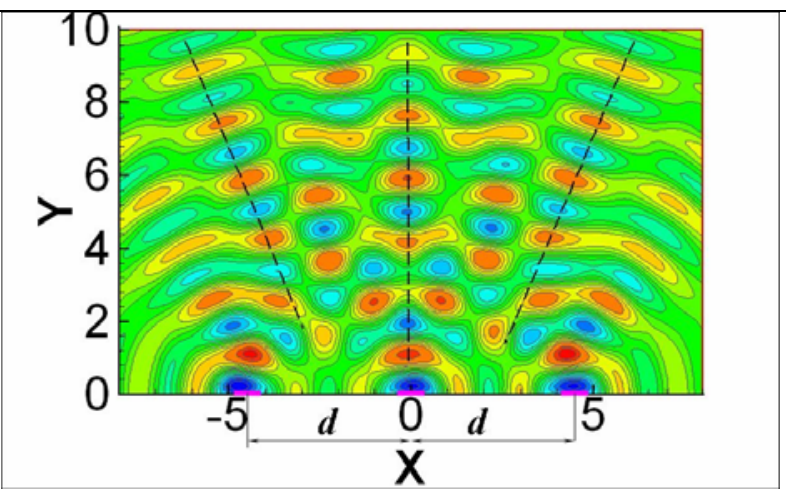

b)

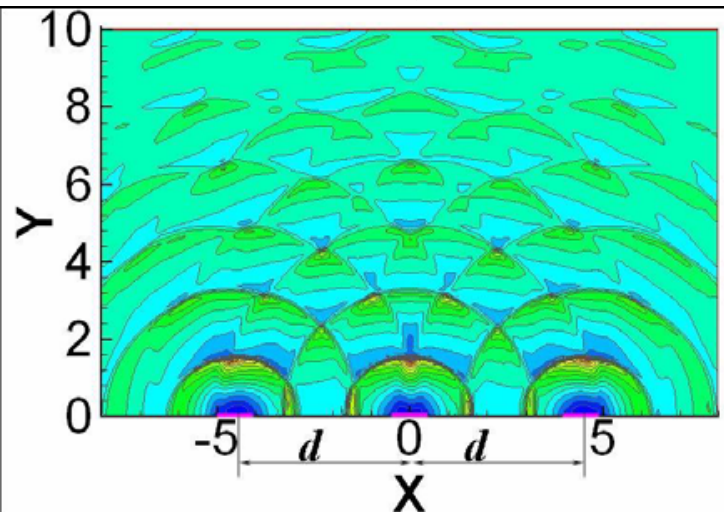

d)

Figure 14. Radiated wave from array of the 3 baffled pistons - contour Plot: $t=12 ; k a=2$,: a) $V_{0}=0.01, d=2$; b) $V_{0}=0.01, d=4.5$; c) $V_{0}=0.5, d=2 ;$ d) $V_{0}=0.5, d=4.5$

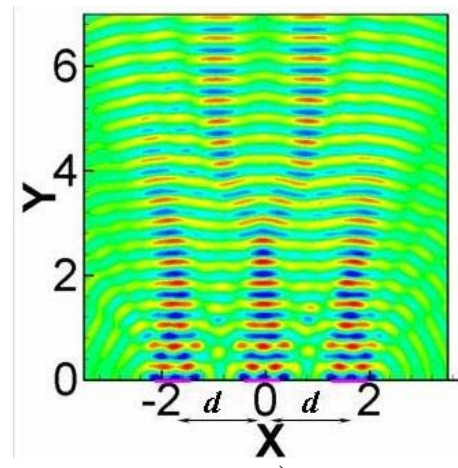

a)

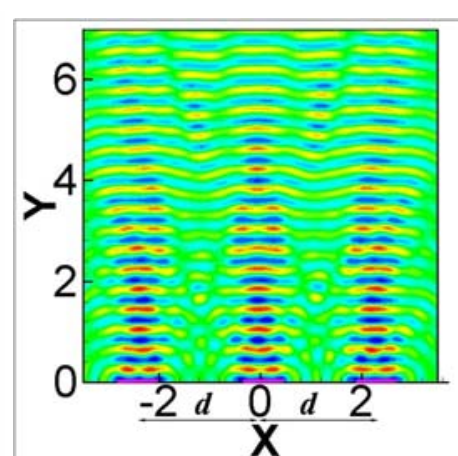

b)

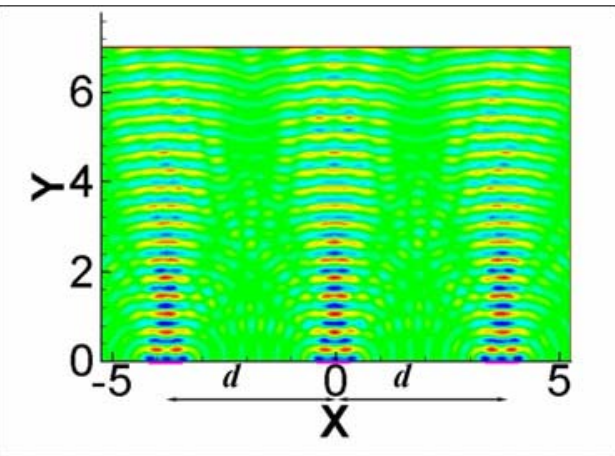

c)

Figure 15. Radiated wave from array of the 3 baffled pistons - contour Plot: $t=12 ; V_{0}=0.01 ; k a=8$; distance between center of the piston (d): a) 1.75 ; b) 2.3 ; c) 3.75 


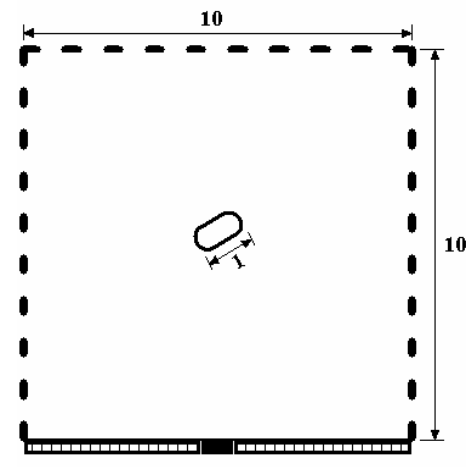

- Outflow BC

Piston

Rigid Baffle

a)

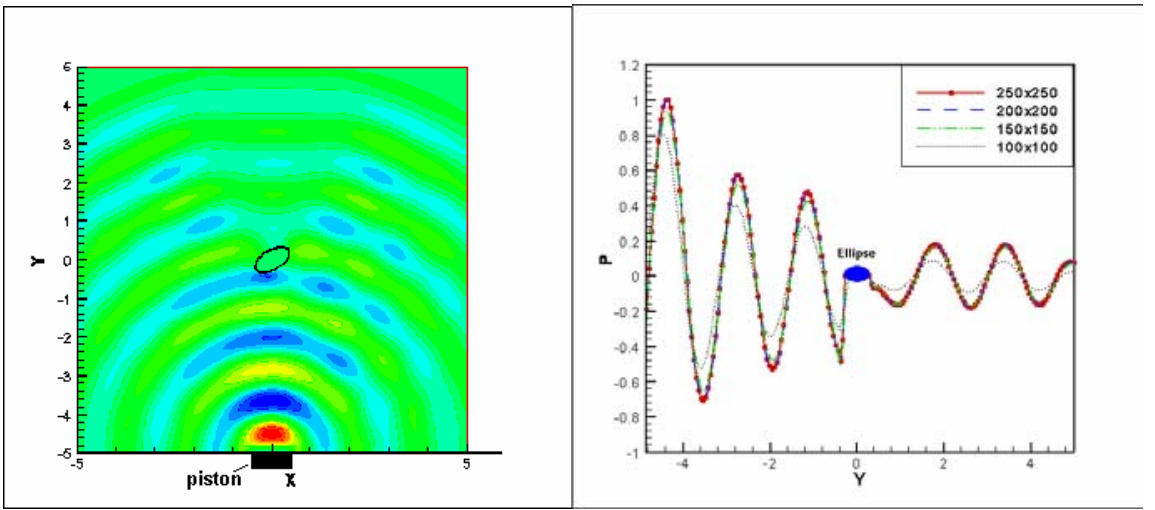

b)

c)

Figure 16. Geometry and pressure distribution of different grid sizes of linear baffled piston with an oblique ellipse, $\omega=4, \mathrm{V0}=1.0, t=10$, a) geometry; b) pressure contour; c) Axial solution

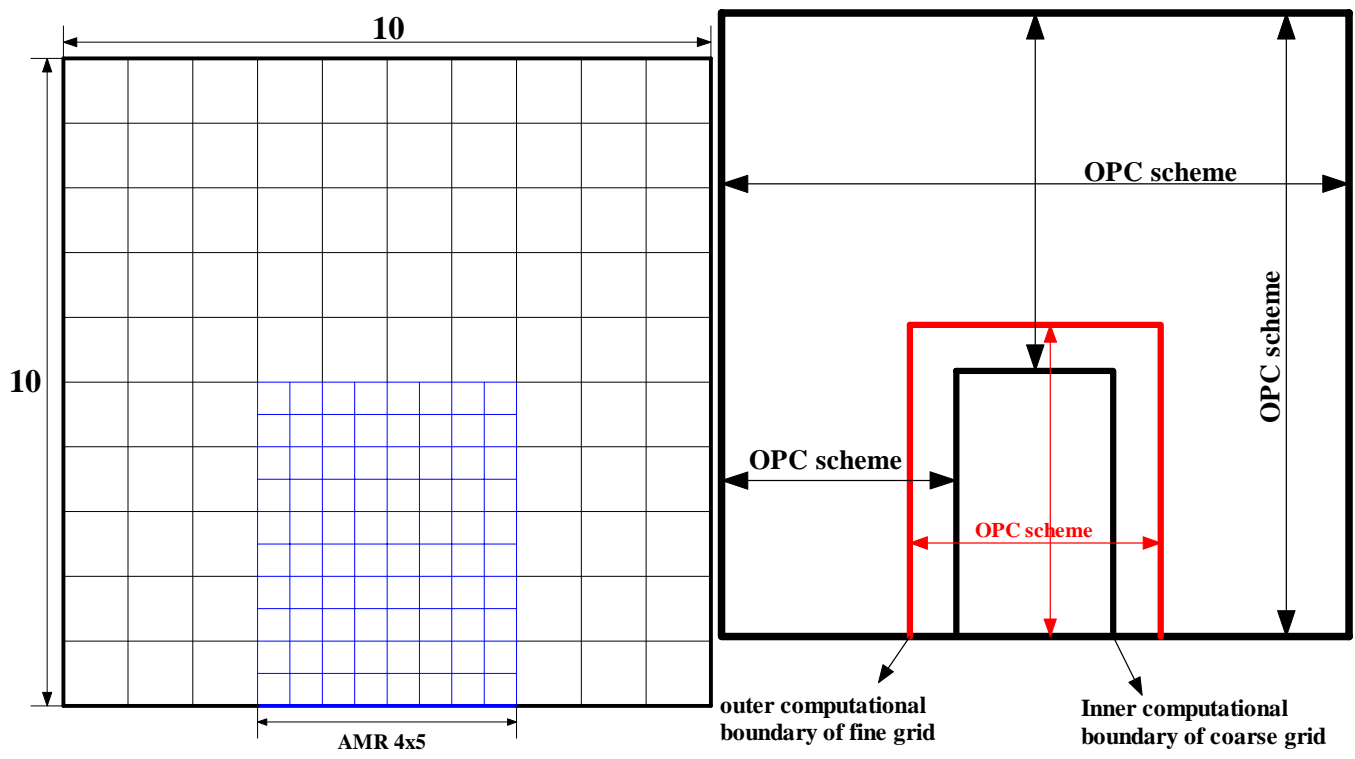

Figure 17. Illustration of geometry of regular and local refined domain and their corresponding computational domains. 


\begin{tabular}{|c|c|c|c|c|c|}
\hline Case & $\begin{array}{l}100 \times 100 \\
\text { (uniform) }\end{array}$ & $\begin{array}{l}135 \times 135 \\
\text { (uniform) }\end{array}$ & $\begin{array}{l}200 \times 200 \\
\text { (uniform) }\end{array}$ & $\begin{array}{l}269 \times 269 \\
\text { (uniform) }\end{array}$ & $\begin{array}{l}300 \times 300 \\
\text { (uniform) }\end{array}$ \\
\hline Cells & 10000 & 18225 & 40000 & 72361 & 90000 \\
\hline Time & 1.0 & 2.44 & 8.54 & 26.33 & 29.58 \\
\hline Error & $14.4 \mathrm{E}-2$ & 11.3E-2 & $9.3 \mathrm{E}-2$ & 8.6E-2 & 8.3E-2 \\
\hline Case & $\begin{array}{c}100 \times 100 \\
(100 \times 100 \text { uniform }+ \\
80 \times 100 \text { refined })\end{array}$ & \multicolumn{2}{|c|}{$\begin{array}{c}200 \times 200 \\
(200 \times 200 \text { uniform }+160 \times 200\end{array}$} & $(300 \times 300$ & $n+240 \times 300$ \\
\hline Cells & $\begin{array}{c}18000 \\
(10000+8000)\end{array}$ & \multicolumn{2}{|c|}{$\begin{array}{c}72000 \\
(40000+32000)\end{array}$} & \multicolumn{2}{|c|}{$(90000+72000)$} \\
\hline Time & 2.84 & \multicolumn{2}{|r|}{26.30} & \multicolumn{2}{|c|}{100.34} \\
\hline Error & $10.1 \mathrm{E}-2$ & \multicolumn{2}{|r|}{$7.8 \mathrm{E}-2$} & \multicolumn{2}{|c|}{$7.3 \mathrm{E}-2$} \\
\hline
\end{tabular}

Table 1 Summary of the computational performance of the grid refinement method.

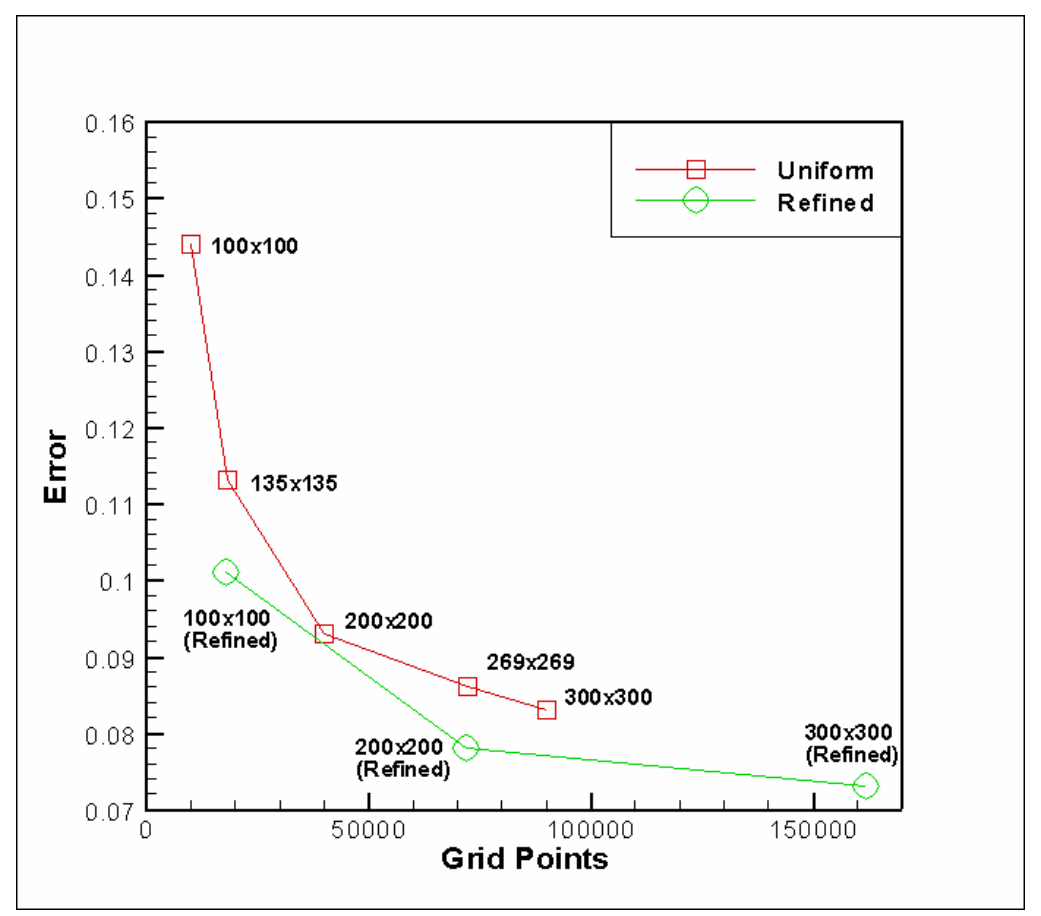

Figure 18. Comparison of errors based on different cases listed in Table 1. 